\title{
Personas mayores y publicidad: Representaciones de género en television
}

\author{
Irene RAMOS SOLER \\ Universidad de Alicante \\ irene.ramos@ua.es \\ Natalia PAPÍ GÁlveZ \\ Universidad de Alicante \\ natalia.p@ua.es
}

Recibido: $27 / 07 / 2012$

Aceptado: 12/10/2012

\begin{abstract}
Resumen
Esta investigación analiza la representación de los mayores en la publicidad televisiva española desde un enfoque de género. Se examina el contenido de 2065 spots de la franja horaria de máxima audiencia durante una semana en 2008 y de cinco cadenas españolas (TVE 1, La 2, Tele 5, Antena 3 y Cuatro). Los resultados revelan que las mujeres mayores están infrarrepresentadas en comparación con los datos demográficos. Igualmente los personajes femeninos y masculinos se muestran con características asociadas al estereotipo de género. Las conclusiones de este estudio están en consonancia con investigaciones previas de otros países. Entre las implicaciones destacan aquellas que atienden a la aplicación de un modelo más igualitario bajo la consideración de sus posibles efectos en el objetivo de la publicidad.

Palabras clave: publicidad; personas mayores; televisión; imagen; género.
\end{abstract}

\section{Elderly and advertising: Gender representations in television}

\begin{abstract}
This study examines the representation of elderly in Spanish television advertising from gender perspective. This inquiry employed a content analysis of 2065 advertisements on prime time television by a week of 2008 from five major spanish channels (TVE 1, La 2, Tele 5, Antena 3 and Cuatro). The results revealed that older females were underrepresented in comparison to demographic reality. Also, female and males characters were showed with attributions of the gender stereotype. Many of these findings are in accordance with previous studies from other parts of the world. Among the implications of the findings, the authors emphasize the study of a type of communication to transmit more egalitarian models under consideration of their potential impact on the goal of advertising.
\end{abstract}

Keywords: advertising, elderly population, television, portrait, gender.

\section{Referencia normalizada}

RAMOS SOLER, Irene y PAPÍ GALVEZ, Natalia (2012): "Personas mayores y publicidad: Representaciones de género en television". Estudios sobre el mensaje periodístico. Vol. 18, núm. especial noviembre, págs.: 753-762. Madrid, Servicio de Publicaciones de la Universidad Complutense.

Sumario: 1. Introducción. 2. Metodología. 3. Resultados; 3.1. La presencia de los mayores en publicidad; 3.2. Diferencias por sexo en la presencia de los mayores en publicidad; 3.3. La representación de las mujeres y hombres mayores en publicidad. 4. Conclusiones. 5. Referencias bibliográficas.

\section{Introducción}

Dos de los criterios que las empresas normalmente consideran cuando evalúan nuevos mercados son su peso económico y demográfico; elementos que hacen a las personas mayores uno de los mercados con mayor futuro. 
En los próximos años se incorporará de forma progresiva a la tercera edad la generación española del baby boom. En nuestro país este fenómeno tuvo lugar entre 1957 y 1977 (Imserso, 2009). Hablamos del maturity boom y del cambio cualitativo experimentado en este mercado respecto a hábitos de consumo, estilos de vida y condiciones socioeconómicas, físicas y psicológicas (Hudson, 2010; Westerhof et al., 2010; Sudbury \& Simcock, 2009; Furlong, 2007; Kohlbacher \& Herstatt, 2008: Thornhill \& Martin, 2007).

Los mayores son, además, grandes consumidores de los medios de comunicación publicitarios aunque, en España, esta publicidad debe considerarse aún incipiente (Ramos-Soler y Carretón Ballester, 2012). Cabe esperar, pues, un incremento sustancial en los próximos años de la presencia de personas mayores en la publicidad y un mayor interés por analizar la imagen.

La gran parte de las investigaciones realizadas sobre mayores y publicidad se han llevado a cabo en EEUU y Reino Unido. Estos estudios coinciden en señalar la escasa presencia de las personas mayores en publicidad, mientras discrepan en la representación, pudiendo ésta ser negativa (o estereotipada) o positiva.

En las revistas dirigidas específicamente a mayores, este colectivo está más presente y su representación es positiva. Aparecen como personas activas y saludables, y con una presencia equilibrada de ambos sexos (i.e. Carrigan \& Szmigin, 1999; Roberts \& Zhou, 1997). La imagen positiva se explicaría por el perfil de la audiencia.

Igualmente, el sentido de la representación de la imagen podría verse condicionado por el producto. A este respecto, las categorías de productos que recurren con más frecuencia al uso de los mayores como modelos publicitarios son los relacionados con la salud y la alimentación; pero también los viajes y el ocio (i.e. Carrigan \& Szmigin, 1998, 2000; Robinson, 1998; Roy \& Harwood, 1997; Balazs, 1995; Tupper, 1995; Zhou \& Chen, 1992).

Así, según la literatura previa, la edad se convierte en un elemento segregador que queda unido a otros tales como el género al afectar en mayor medida a las mujeres (i.e. Carrigan \& Szmigin, 1998, 1999, 2000; Roberts \& Zhou, 1997; Roy \& Harwood, 1997; Kaid \& Garner, 1995; Peterson, 1992, 1995).Esta forma de tratar al mercado maduro, contribuye por un lado, a que las empresas pierdan ventas potenciales, y por otro, a que una amplia sección de la sociedad esté siendo considerada de una manera muy pobre como consumidores.

En España, empiezan a publicarse trabajos que analizan la imagen del mayor en la publicidad (Ramos-Soler y Carretón Ballester, 2012; Ramos-Soler, 2007; Sánchez Vera y Bódalo Lozano, 2000; Freixas, 1998) pero pocas investigaciones académicas profundizan en la relación entre género y edad madura. La escasez de investigaciones contrasta con la proliferación de estudios sobre la imagen de los últimos años.

Por ello, esta investigación se ocupa de la presencia y representación de los mayores en la publicidad española desde un enfoque de género. En concreto persigue:

1. Valorar la presencia de los mayores en la publicidad según la realidad demográfica.

2. Comparar la presencia por sexo a la luz de los datos poblacionales.

3. Averiguar si la representación responde al estereotipo de género. 


\section{Metodología}

Se contabilizaron las inserciones publicitarias y se analizó el contenido de las piezas emitidas en TVE1, La 2, Tele 5, Antena 3 y Cuatro. Se seleccionó una semana al azar de 2008 (10-16 noviembre) grabándose la programación televisiva en prime-time (20.30-22.30). El contraste empírico se realizó sobre una muestra final de 2065 spots. Aparece población mayor en el 25\% de los anuncios (513 spots).

Se contaron las repeticiones de anuncios (y por tanto las inserciones) porque esta muestra refleja mejor el propósito (y el esfuerzo) publicitario. Se eligió la televisión como medio de comunicación de mayor penetración en las personas de 65 y más años. En cuanto al periodo, la semana es la unidad temporal para la programación, por lo que se considera representativa del resto en un periodo de tiempo razonable.

El año 2008 se eligió como inicio del periodo en el que se advierte la presencia de mayores en la publicidad como posible respuesta al advenimiento del maturity boom, pues en este año la primera generación del baby boom (nacida en 1957) tenía 51 años. Así, se es coherente con los criterios de inclusión establecidos en el análisis de contenido al considerar un margen de edad más amplio, siendo "mayores" aquellas personas que aparentaban tener en el contexto de la historia publicitaria más de 50 años. Este criterio se justifica tanto por las estrategias llevadas a cabo por la publicidad que tratan de huir de los modelos excesivamente realistas (y transmiten más juventud) como por las particularidades del análisis de contenido, que implica una codificación de la apariencia a través de la percepción de los jueces. El estudio tendería a la sobrerrepresentación frente al criterio demográfico (65 y más años). Por lo que si se llegara a la conclusión de que la publicidad de mayores es escasa, como han demostrado otros estudios en otros países, sería pese a este sesgo que favorece su inclusión.

Además, se incluyeron aquellos spots en los que los personajes: 1. aparecen en un contexto de retiro, 2. tienen pelo gris o blanco y/o 3. arrugas, 4. son padres de hijos de mediana edad y/o 5. se muestran nietos, 6 . usan prótesis ambulatorias o 7 . se hace mención expresa. En cambio, no se contabilizaron partes del cuerpo o cuerpos sin cara.

El cuestionario se elaboró atendiendo a la literatura y a los objetivos del estudio. En concreto, para el contexto y el escenario se siguió a Fernández y Martínez (1999) y a Carmona (2005). Las categorías fueron:

- Sexo (mujeres y hombres).

- Importancia del rol (protagonista, secundario, de fondo).

- Papel desempeñado (padre-madre, abuelo/a, esposo/a, otro-familiar, amigo/a, compañero/a-trabajo, conocido/a, otros).

- Estilo del vestuario (deportivo, elegante-serio-profesional, casual, descuidadohortero-raro, tradicional, no se muestra).

- Imagen física-social (bien-parecido, poco-agraciado, saludable, enfermizo-dependiente, divertido, serio, feliz, triste, sociable, antisocial, activo, pasivo, modernoactual, antiguo-tradicional-conservador, bueno, malo, responsable-organizado, irresponsable-desorganizado, lúcido-intelectual, confuso-despistado).

- Productos, según distribución de sectores de Infoadex.

- El contexto general (rural, urbano o plano neutro). 
- El escenario (hogar-doméstico, lúdico-ocio, trabajo-laboral, deportivo, educativo-cultural, religioso, sanitario-salud, centro de mayores, sin escenario, vía pública).

Dos jueces independientes codificaron todos los anuncios. El índice del grado de acuerdo resultó 0,91 . Se procesó la información con SPSS y SPAD.

Se aplicó un análisis descriptivo-multivariable, (caracterización estadística) que permitió conocer las características más sobresalientes (estadísticamente significativas) de los hombres en relación a las mujeres, y viceversa.

\section{Resultados}

\subsection{La presencia de los mayores en publicidad}

En el $25 \%$ de los spots aparecían personas de $50+$ años, mientras que en ese mismo año (2008) suponían el 33\% de la población. Sin embargo, si se atiende al segmento poblacional que se ajusta a la definición más utilizada para hacer referencia a personas mayores $(65+)$ la presencia en publicidad era superior en 8 puntos.

\subsection{Diferencias por sexo en la presencia de los mayores en publicidad}

Los modelos masculinos predominaban en los anuncios (tabla 1). La presencia de los hombres llegaba a superar en 42 puntos a la de las mujeres. Estos datos contrastaban con las tasas poblacionales (el $54 \%$ de población mayor de 50 años son mujeres; el $58 \%$ de la población mayor de 65 años son mujeres).

Tabla 1. Tasas de anuncios de mayores por sexo del modelo. Fuente: Elaboración propia.

\begin{tabular}{|l|c|c|c|c|}
\hline & $\begin{array}{c}\text { Hombres } \\
\text { mayores }\end{array}$ & $\begin{array}{c}\text { Mujeres } \\
\text { mayores }\end{array}$ & Total & $\begin{array}{c}\text { Total } \\
\text { anuncios }\end{array}$ \\
\hline $\mathrm{N}$ & 364 & 149 & 513 & 2065 \\
\hline$\%$ anuncios mayores & $71 \%$ & $29 \%$ & $100 \%$ & - \\
\hline$\%$ total anuncios & $18 \%$ & $7 \%$ & - & $25 \%$ \\
\hline
\end{tabular}

\subsection{La representación de las mujeres y hombres mayores en publicidad}

Los anuncios en los que el mayor que aparece era mujer destacaban por ofrecer una imagen antigua, tradicional, conservadora, buena, bondadosa y feliz. El contexto general era rural, con un escenario hogareño o doméstico. El rol desempeñado predominante era el de madre o abuela, con papeles secundarios y con productos farmacéuticos, de óptica o de alimentación (tabla 2).

Tabla 2. Caracterización de las mujeres mayores (Spots 149- Porcentaje 29.04). Variables nominales. Fuente: Elaboración propia. Nota1: orden según el nivel de significación (PROBA.) Nota2: cuando hay más de un personaje, se recogen los datos de aquel al que se le atribuye el papel principal. 


\begin{tabular}{|l|l|c|c|c|c|}
\hline VARIABLE & MODALIDAD & $\begin{array}{c}\text { \% } \\
\text { MOD/CLASE }\end{array}$ & $\begin{array}{c}\text { \% } \\
\text { CLAAOMOD }\end{array}$ & PROBA. & n \\
\hline Producto que se anuncia & Alimentación & 32.89 & 83.05 & 0.000 & 59 \\
\hline $\begin{array}{l}\text { Contexto general en } \\
\text { que aparece el mayor }\end{array}$ & Rural & 20.13 & 73.17 & 0.000 & 41 \\
\hline Estilo de vestuario & Tradicional & 36.24 & 47.79 & 0.000 & 113 \\
\hline Rol desempeñado & Madre & 19.46 & 58 & 0.000 & 50 \\
\hline Escenario & Hogar/doméstico & 46.31 & 41.32 & 0.000 & 167 \\
\hline Rol desempeñado & Abuela & 15.44 & 60.53 & 0.000 & 38 \\
\hline Imagen & $\begin{array}{l}\text { Antigua, tradicional } \\
\text { conservadora }\end{array}$ & 44.30 & 39.76 & 0.000 & 166 \\
\hline Estilo de vestuario & No se muestra vestuario & 8.05 & 70.59 & 0.000 & 17 \\
\hline Imagen & Bueno, bondadoso & 10.07 & 57.69 & 0.002 & 26 \\
\hline $\begin{array}{l}\text { Magnitud o importancia } \\
\text { del papel del mayor }\end{array}$ & Secundario & 40.27 & 38.22 & 0.002 & 157 \\
\hline Imagen & Feliz & 47.65 & 35.68 & 0.006 & 199 \\
\hline Producto que se anuncia & $\begin{array}{l}\text { Productos farmaceúticos } \\
\text { y óptica }\end{array}$ & 8.72 & 54.17 & 0.007 & 24 \\
\hline Estilo de vestuario & Informal pero cuidado & 22.82 & 40.48 & 0.009 & 84 \\
\hline
\end{tabular}

Los anuncios en los que el mayor era hombre destacaban por ofrecer una imagen de éste como una persona activa, saludable, independiente y responsable. El contexto en el que aparecía era urbano. El rol desempeñado era de compañero de trabajo; con vestuario deportivo o profesional; con apariciones fugaces y en anuncios de tecnología, comunicación, finanzas o electrónica (tabla 3).

Tabla 3. Caracterización de los hombres mayores (Spots 364-Porcentaje 70.96). Variables nominales. Fuente: Elaboración propia. Nota1: orden según el nivel de significación (PROBA.). Nota2: cuando hay más de un personaje, se recogen los datos de aquel al que se le atribuye el papel principal.

\begin{tabular}{|l|l|c|c|c|c|}
\hline VARIABLE & MODALIDAD & $\begin{array}{c}\text { \% } \\
\text { MOD/CLASE }\end{array}$ & $\begin{array}{c}\text { \% } \\
\text { CLA/MOD }\end{array}$ & PROBA. & n \\
\hline Producto anunciado & $\begin{array}{l}\text { Telefonía y aparatos de } \\
\text { comunicación }\end{array}$ & 16.21 & 98.33 & 0.000 & 60 \\
\hline Rol desempeñado & Otros & 40.11 & 85.38 & 0.000 & 171 \\
\hline $\begin{array}{l}\text { Contexto general en el } \\
\text { que aparece el mayor }\end{array}$ & Urbano & 80.77 & 76.96 & 0.000 & 382 \\
\hline Escenario & Vía pública & 28.57 & 86.67 & 0.000 & 120 \\
\hline Estilo de vestuario & $\begin{array}{l}\text { Elegante, serio y profesio- } \\
\text { nal }\end{array}$ & 44.23 & 82.14 & 0.000 & 196 \\
\hline
\end{tabular}




\begin{tabular}{|l|l|c|c|c|c|}
\hline VARIABLE & MODALIDAD & $\begin{array}{c}\text { \% } \\
\text { MOD/CLASE }\end{array}$ & $\begin{array}{c}\text { \% } \\
\text { CLAOMOD }\end{array}$ & PROBA. & n \\
\hline $\begin{array}{l}\text { Magnitud o importancia } \\
\text { de papel desempeñado }\end{array}$ & Aparición fugaz & 24.45 & 87.25 & 0.000 & 102 \\
\hline Estilo de vestuario & Sin definir & 9.89 & 97.30 & 0.000 & 37 \\
\hline Imagen & Organizado/responsable & 21.43 & 86.67 & 0.000 & 90 \\
\hline Rol desempeñado & Compañero de trabajo & 9.07 & 94.29 & 0.001 & 35 \\
\hline Imagen & $\begin{array}{l}\text { Saludable, independiente } \\
\text { físicamente }\end{array}$ & 50 & 77.45 & 0.002 & 235 \\
\hline Escenario & Lúdico/ocio & 9.62 & 89.74 & 0.004 & 39 \\
\hline Estilo de vestuario & Deportivo & 4.40 & 100 & 0.004 & 16 \\
\hline Imagen & Divertida & 23.35 & 81.73 & 0.004 & 104 \\
\hline Canal de TV & Cuatro & 14.29 & 85.25 & 0.005 & 61 \\
\hline Producto que se anuncia & Finanzas, seguros, loterías & 17.86 & 83.33 & 0.005 & 78 \\
\hline Imagen & Persona activa & 53.57 & 76.17 & 0.006 & 256 \\
\hline Producto que se anuncia & $\begin{array}{l}\text { Electrodomésticos y acce- } \\
\text { sorios hogar }\end{array}$ & 3.85 & 100 & 0.008 & 14 \\
\hline Producto que se anuncia & $\begin{array}{l}\text { Medios de comunicación } \\
\text { y artículos de oficina }\end{array}$ & 27.75 & 79.53 & 0.009 & 127 \\
\hline
\end{tabular}

En cuanto a la posición que ocupaban en la historia publicitaria, en el $70 \%$ de los spots de mayores era el hombre quien realizaba el papel principal, frente al $30 \%$ de las mujeres. Este menor peso de la mujer prevalecía en todos los roles (tabla 4, \% por fila). Cuando se mostraban mujeres éstas solían ocupar el rol de protagonista o de secundario en mayor medida que cuando se mostraban hombres (tabla 4, \% por columnas) aunque las diferencias entre sexos eran bastante más profundas en los secundarios $\mathrm{y}$ fugaces.

El alto porcentaje de mujeres que se ubicaban en un papel secundario llevaba a considerar que había menos anuncios en los que éstas aparecían solas, como confirmó el menor porcentaje de "mujeres" en la tabla 5.

Tabla 4. Rol desempeñado por la persona mayor en la historia publicitaria: distribución por sexo. Fuente: Elaboración propia. Nota: col. \% por columnas; fil. \% por filas.

\begin{tabular}{|l|c|c|c|}
\hline & Hombres mayores & Mujeres mayores & Total \\
\hline Papel protagonista & $178(\mathrm{Col} .49 \%$, Fil. $70 \%)$ & $76($ Col. $51 \%$, Fil. 30\%) & $254($ Fil. 100\%) \\
\hline Papel secundario & $97($ Col. $27 \%$, Fil. $62 \%)$ & $60($ Col. $40 \%$, Fil. $38 \%)$ & $157($ Fil. $100 \%)$ \\
\hline Aparición fugaz & $89($ Col. $24 \%$, Fil. $87 \%)$ & $13($ Col. $9 \%$, Fil. $13 \%)$ & $102($ Fil. $100 \%)$ \\
\hline Total & $364($ Col. $100 \%)$ & $149($ Col. $100 \%)$ & 513 \\
\hline
\end{tabular}


Tabla 5. Tasas de anuncios de mayores por sexo según tipo de presencia. Fuente: Elaboración propia.

\begin{tabular}{|l|c|c|c|c|c|}
\hline & Hombres & Mujeres & Presencia conjunta & Total & Total anuncios \\
\hline $\mathrm{N}$ & 303 & 86 & 124 & 513 & 2065 \\
\hline$\%$ anuncios mayores & $59 \%$ & $17 \%$ & $24 \%$ & $100 \%$ & - \\
\hline$\%$ total anuncios & $14,7 \%$ & $4,2 \%$ & $6 \%$ & - & $25 \%$ \\
\hline
\end{tabular}

\section{Conclusiones}

Este estudio es uno de los primeros en profundizar en la representación de las personas mayores en publicidad desde una perspectiva de género en España. Desemboca en dos principales conclusiones que responden a los objetivos iniciales: existe un profundo desequilibrio entre presencia publicitaria y peso demográfico por sexo; y se utiliza el estereotipo de género para representar a las mujeres y los hombres de esta edad. A estas dos se le añade el diferente valor de las mujeres y los hombres cuando se observa la posición de los mismos en la historia publicitaria que contribuye, en última instancia, a reforzar igualmente el estereotipo.

Así, para las mujeres mayores la distancia entre su presencia en la publicidad y la realidad demográfica es siempre negativa en cualquiera de los dos grupos de edad $(50+$ ó $65+)$ llegando a ser de hasta 29 puntos. Esta distancia significaría otorgar la mitad del peso a las mujeres, a favor de los hombres. Es más, los datos apuntan a que cuando existe infrarrepresentación del grupo etario en su conjunto se debe fundamentalmente a la invisibilidad de las mujeres.

En cuanto a los estereotipos, de acuerdo con el análisis de características, a la mujer se le sigue ubicando en la esfera doméstica y al hombre en la esfera pública; mostrando una imagen entroncada en la división sexual del trabajo que define los roles de género más fundamentales. La mujer es madre y/o abuela y el hombre es compañero de trabajo. El escenario para la mujer es doméstico y, en cambio, para el hombre es externo a lo doméstico. A la mujer se le presenta con bondad, característica atribuible a ser madre y/o abuela. El hombre, en cambio, es activo, saludable, profesional. Y todas estas atribuciones están en consonancia con el tipo de producto anunciado. Para la mujer, los productos sobresalientes son la alimentación o los relacionados con la salud, propios del cuidado a la familia. Para los hombres, la tecnología o los medios de comunicación, que son intelectuales, en estrecha conexión con el ejercicio profesional y con la esfera pública.

Finalmente, en cuanto a la valoración, las mujeres ocupan roles secundarios en mayor medida que los hombres. Este resultado queda reforzado por la menor cantidad de anuncios en los que la mujer aparece sola, ya que una posición secundaria precisa de una referencia primaria por definición. Además, los roles que le son atribuidos a la mujer (madre/abuela) precisan de una relación con otra persona de la propia familia (hijos/nietos) para ser definidos. Por tanto, incluso cuando la mujer aparece sola en el anuncio, el papel de madre o abuela refuerza, a través de la imagen proyectada, el rol que la sociedad adjudica a la mujer mayor (en el ámbito familiar), con todas las implicaciones conocidas, que han sido explicadas por los estudios de género. 
La aparición fugaz en los hombres no debe ser confundida con una posición menos ventajosa. Tiene una función de representación hacia el grupo poblacional, sin operar como prescriptor, por lo que no se establece una jerarquía como la que implica el personaje secundario. El hecho de que se utilicen hombres en apariciones fugaces atiende a que la generalización no es neutra, sino masculina. Es decir, la representación del grupo (a través de la generalización) se hace por medio de un varón, lo que favorece la invisibilidad de la mujer.

Estas conclusiones conducen a confirmar la existencia de un modelo sexuado de publicidad que responde al sistema de género. Entre las implicaciones futuras destacan aquellas que atienden al estudio de la aplicación de un modelo más igualitario, bajo la consideración de sus posibles efectos en el objetivo de la publicidad; pues la publicidad utiliza los estereotipos para transmitir eficazmente el mensaje pretendido, el cual debe satisfacer los objetivos propuestos. Por lo que la cuestión es si puede conseguir lo mismo sin recurrir a los estereotipos de género.

\section{Referencias bibliográficas}

BALAZS, Anne (1995): "The use and image of mature adults in health care advertising (1954-1989). Health Marketing Quarterly, 12, n. 3. Routledge, pp.13-26.

CARMONA, Ramón (2005): Cómo se comenta un texto filmico. Madrid, Paidós.

CARRIGAN, Marylyn \& SZMIGIN, Isabelle (1998): "The usage and portrayal of the older models in contemporary consumer advertising". Journal of Marketing Practice: Applied Marketing Science, 4, n. 8, MCB University Press, pp. 231-248.

CARRIGAN, Marylyn \& SZMIGIN, Isabelle (1999): "The portrayal of older characters in magazine advertising". Journal of Marketing Practice: Applied Marketing Sciencie, 5, n. 6/7/8. MBC University Press, pp. 248-261.

CARRIGAN, Marylyn \& SZMIGIN, Isabelle (2000): "Advertising in an Ageing society”. Ageing and Society, n. 20. Cambridge University Press, pp. 217-233.

FERNÁNDEZ, Federico y MARTÍNEZ, José (1999): Manual básico de lenguaje y narrativa audiovisual. Barcelona, Paidós Papeles de Comunicación 22.

FREIXAS, Anna (1998): "El doble estándar del envejecimiento en la publicidad televisiva". Comunicación y Cultura, $\mathrm{n}^{\circ} 3$. Madrid, Fundación infancia y aprendizaje, pp 29-40.

FURLONG, Mary (2007): Turning silver into gold. How to Profit in the New Boomer Marketplace. New Jersey (EEUU), Press Financial Times.

HUDSON, Simon (2010): "Wooing zoomers: marketing to the mature traveler". Marketing Intelligence \& Planning, 28, n. 4. EmeraldEarlycite, pp. 444-461.

IMSERSO (2009): Informe 2008. Las personas mayores en España. Colección Documentos, Serie Documentos Estadísticos, $n^{\circ} 22019$. Madrid, IMSERSO.

KAID, Lynda Lee \& GARNER, Jane (1995): "Political advertising and the elderly" en NUSSBAUM, John \& COUPLAN, Justine: Handbook of Communication and 
Aging Research. Abingdon, United Kingdom, Lawrence Erlbaum Associates Publishers, pp.343-357.

KOHLBACHER, Florian \& HERSTATT, Cornelius (2008): The silver market phenomenon. Berlin (Germany): Springer.

PETERSON, Robin (1992): “The depiction of senior citizens in magazine advertisements: a content analysis". Journal of business ethics, n. 11. Springer, pp.701-706.

PETERSON, Robin (1995): "The portrayal of senior citizens by banks in newspaper advertisements: a content analysis". Journal of Professional Services Marketing, 12, n. 2. Routledge, pp. 95-106.

RAMOS-SOLER, Irene (2007): El estilo de vida de los mayores y la publicidad. Barcelona: Fundación "La Caixa".

RAMOS-SOLER, Irene y CARRETÓN BALLESTER, M Marmen (2012): "Presencia y representación de las personas mayores en la publicidad televisiva: El caso español". Revista Española de Geriatría y Gerontología, 47, nº 2. Madrid, Sociedad española de geriatría y Gerontología, pp. 55-61.

ROBERTS, Scott \& ZHOU, Nan (1997): "The 50 and Older Characters in the advertisements of Modern Maturity: Growing Older, Getting Better?". The Journal of Applied Gerontology, 16, n. 2. Sage Publications pp.208-220.

ROBINSON, Thomas (1998): Portraying older people in advertising. London, Garland Publishing.

ROY, Abhik \& HARWOOD, Jake (1997): "Underrepresented, Positively Portrayed: Older Adults in Televisión Commercials". Journal of Applied Communication Research, n. 25, Routledge and National Communication Association, pp.39-56.

SÁNCHEZ VERA, Pedro y BÓDALO LOZANO, Esther (2000): "Presencia y ausencia de los mayores en la publicidad televisiva". Revista del Ministerio de Trabajo y Asuntos Sociales, n. 25. Madrid, Ministerio de Trabajo y Asuntos Sociales, pp.19-25.

SUDBURY, Lynn \& SIMCOCK, Peter (2009): "Understanding older consumers through cognitive age and the list of values: A U.K.-based perspective". Psychology and Marketing, 26, n. 1. AMA, pp. 22-38.

THORNHILL, Matt \& MARTIN, John (2007): Boomer Consumer: ten rules for marketing to America's largest, wealthiest and most influential group. Great Falls Virginia (USA), LINX Corp.

TUPPER, Meredith (1995): The representation of elderly persons in primetime televisión advertising. Florida, University of South Florida.

WESTERHOF, Gerben; HARINK, Karolien; VAN SELM, Martine; STRICL, Madelijn; VAN BAAREN, Rick (2010): "Filling a missing link: the influence of portrayals of older characters in television commercials on the memory performance of older adults". Ageing and Society, 30, n. 5, British Society of Gerontology, pp. 897-912. 
ZHOU, Nan \& CHEN, Mervin (1992): "Marginal life after 49: a preliminary study of the portrayal of older people in Canadian consumer magazine advertising". International Journal of Advertising, n. 11. The Advertising Association by Warc. pp.343-354.

\section{Irene RAMOS SOLER}

Irene.Ramos@ua.es

Dpto. Comunicación y Psicología Social. Facultad de Ciencias Económicas y Empresariales. Edificio de Ciencias Sociales. Universidad de Alicante. Campus de San Vicente del Raspeig. Apartado de correos 99

Universidad de Alicante

Directora del Grupo de Investigación "Comunicación y Públicos específicos"

Contratada doctora del dpto. Comunicación y Psicología Social.

\section{Natalia PAPÍ GÁLVEZ}

natalia.p@ua.es

Dpto. Comunicación y Psicología Social. Facultad de Ciencias Económicas y Empresariales. Edificio de Ciencias Sociales. Universidad de Alicante. Campus de San Vicente del Raspeig. Apartado de correos 99

Universidad de Alicante

Directora del Grupo E-COM (Estudios de Comunicación estratégica) de la UA.

Titular de universidad del dpto. Comunicación y Psicología Social. 